\title{
NADPH-Independent Inactivation of CYP2B6 and NADPH-Dependent Inactivation of CYP3A4/5 by PBD: Potential Implication for Assessing Covalent Modulators for Time-Dependent Inhibition ${ }^{\circledR}$
}

\author{
Mika Kosaka, Donglu Zhang, Simon Wong, and Zhengyin Yan \\ Drug Metabolism and Pharmacokinetics, Genentech, South San Francisco, California
}

Received February 5, 2020; accepted May 13, 2020

\section{ABSTRACT}

Pyrrolo[2,1-c][1,4]benzodiazepine dimer (PBD) has shown broad antitumor properties and potential as a therapeutic agent for cancers. During a routine drug-drug interaction assessment, it was found that PBD is a reversible inhibitor of CYP2C8 $\left(I_{50}=1.1\right.$ $\mu \mathrm{M})$ but not CYP1A2, 2B6, 2C9, 2C19, 2D6, or 3A4/5. Additionally, PBD is a classic time-dependent inhibition (TDI) of CYP3A4/5, with $>30$-fold shift in $\mathrm{IC}_{50}$ after a preincubation with NADPH. All other CYPs tested did not show evidence for TDI, but potent inhibition of CYP2B6 $\left(I_{50}=1.5 \mu \mathrm{M}\right)$ was observed after a preincubation with or without (w/wo) NADPH, which was an unexpected observation given the fact that no inhibition was observed in the direct inhibition assay. No other CYP isoforms were susceptible to this apparent non-NADPH-dependent inhibition, suggesting that PBD may selectively inactivate CYP2B6 without metabolic activation. The washing of the human liver microsome pellet after incubation with PBD did not fully recover CYP2B6 activity, indicating that PBD is covalently bound to CYP2B6, leading to inactivation of the enzyme. To further investigate the mechanism of NADPH-independent inhibition, the IC $_{50}$ shift was determined for several PBD analogs, and it was found that the compounds without both reactive imines did not show NADPHindependent inhibition of CYP2B6, implying that NADPHindependent inactivation was likely caused by direct covalent binding of PBD to the enzyme in a highly structure-specific manner. These data clearly highlight the need to assess direct and time-dependent inhibition w/wo NADPH to adequately characterize the in vitro CYP inhibitory properties of drug candidates with reactive moieties.

\section{SIGNIFICANCE STATEMENT}

We described a very unique in vitro CYP inhibition profile of pyrrolo [2,1-c][1,4]benzodiazepine dimer as a potent reversible CYP2C8 inhibitor, an NADPH-dependent CYP3A4/5 time-dependent inhibition (TDI) inhibitor, and an NADPH-independent CYP2B6 TDI inhibitor, and inhibition of CYPs occurs through three distinct mechanisms: reversible drug-enzyme binding, enzyme inactivation via bioactivation, and enzyme inactivation by covalent binding via chemical reactions. Our results suggest that, for compounds with reactive functional moieties, false positives can be reported when the conventional TDI assay is utilized.

\section{Introduction}

The pyrrolo[2,1-c][1,4]benzodiazepines (PBDs) are an important class of DNA alkylators that bind to the DNA minor groove in a sequence-selective manner to form a covalent aminal bond between their $\mathrm{C}_{11}$-position and the $\mathrm{C}_{2}-\mathrm{NH}_{2}$ groups of guanine bases of the targeted double-stranded DNA region (Fig. 1A) (Hartley, 2011; Mantaj et al., 2017). Compared with monomeric PBDs, PBD dimers contain two alkylating imines that, after binding to the DNA minor groove, can form intrastrand/interstrand DNA crosslinks, leading to formation of a more stable PBD-DNA complex compared with monoalkylated adducts (Fig. 1B). The PBD dimer molecular structure can influence DNA sequence recognition given that such compounds contain a chiral $\mathrm{C}_{11} \mathrm{a}(\mathrm{S})$-center that forms an appropriate conformation to fit in the minor groove of DNA, resulting in superior alkylating potency compared with PBD monomers.

\section{Conflict of Interest: Authors have no conflict of interest to declare.}

https://doi.org/10.1124/dmd.120.090878.

$\$$ This article has supplemental material available at dmd.aspetjournals.org.
Through covalent bonding to DNA, PBDs have been shown to exert a number of biologic effects in cells, including DNA strand breakage (Reid et al., 2011), inhibition of DNA-processing enzymes such as endonuclease (Puvvada et al., 1993), RNA polymerase (Puvvada et al., 1997) and ligase (Shameem et al., 2015), and specific transcriptional factors (Kotecha et al., 2008). Consequently, PBDs have been investigated for their broad antitumor activity and as potential therapeutic agents against a variety of oncology targets, and several PBD dimers, such as SJG-136 and DSB-120, have been advanced to clinic for cancer treatments, including leukemia and ovarian cancer (Martin et al., 2005; Cipolla et al., 2009; Hartley, 2011).

Apparently, cellular cytotoxicity of both PBD monomers and dimers is not highly target-selective, and their inherited off-target activity can potentially result in nonselective cytotoxicity, which severely limits their therapeutic potential as anticancer agents (Mantaj et al., 2017). To mitigate the nonselective cytotoxicity, PBD dimers have recently been attached as a cytotoxic warhead to tumor-specific antibodies via a selfimmolative linker to the $\mathrm{N}_{10}$ nitrogen to generate antibody-drug conjugates (ADCs) (Zhang et al., 2019). As a result, PBD dimers can

ABBREVIATIONS: ACN, acetonitrile; ADC, antibody-drug conjugate; DDI, drug-drug interaction; GSH, glutathione; HLM, human liver microsome; $\mathrm{KPi}$, potassium phosphate; LC-MS/MS, liquid chromatography tandem mass spectrometry; PBD, pyrrolo[2,1-c][1,4]benzodiazepine dimer; rCYP, recombinant CYP; TDI, time-dependent inhibition; w/wo, with or without. 
A<smiles>C=C1C[C@H]2C=Nc3cc(OC)c(OC)cc3C(=O)N2C1</smiles>

Pyrrolo[2,1-c][1,4]benzodiazepine
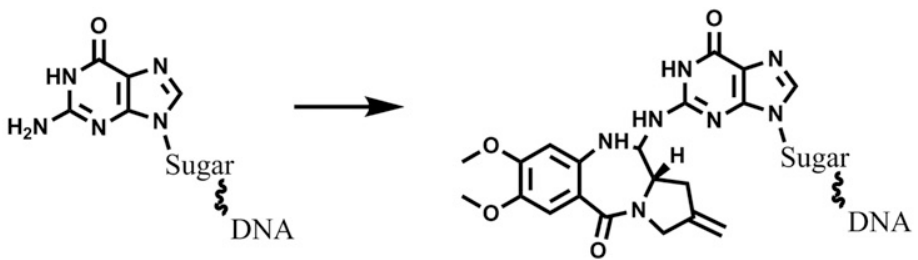

Guanine base

B

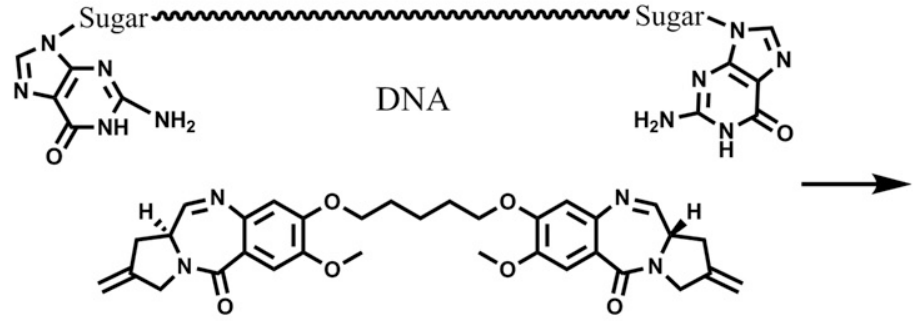

PBD dimer

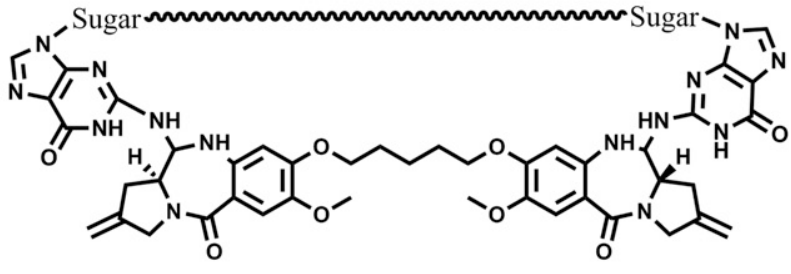

PBD-cross linking

Fig. 1. Formation of the aminal bond between guanine and PBD monomer (top) and dimer (bottom), respectively.

be delivered by antigen-specific monoclonal antibodies specifically to the targeted tumor cells. Once bound to the tumor cells, ADCs can then be internalized via endocytosis and subsequently degraded to generate the $\mathrm{C}_{11}-\mathrm{N}_{10}$ imine group upon linker cleavage, releasing PBD dimers to exert their cancer cell-killing activity (Gregson et al., 2004; Hartley et al., 2004). This immunologic targeting strategy has resulted in several ADCs advancing to clinical development (Zammarchi et al., 2018; Phillips et al., 2019; Zheng et al., 2019).

Clinical interest in developing ADCs as potential cancer therapeutics led us to investigate PBD dimer (referred to as PBD below) for its potential to inhibit common human CYPs in vitro and to pursue extrapolation of such findings to clinical drug-drug interaction (DDI) potential. As a routine experimental assessment, we evaluated pyrrolo $[2,1-c][1,4]$ benzodiazepine, a commonly used PBD warhead for inhibition of seven common cytochrome P450 enzymes, including CYP1A2, CYP2B6, CYP2C8, CYP2C9, CYP2C19, CYP2D6, and CYP4/5 in human liver microsomes (HLMs). Also, $\mathrm{IC}_{50}$ shift assay was conducted to determine whether PBD is a time-dependent inactivator of those common CYP enzymes. Additionally, several PBD analogs were studied to investigate the effect of the reactive moieties on the timedependent inhibition (TDI) of CYP2B6. Since PBDs represent a novel class of drug molecules with chemically reactive moieties, the outcomes of the present investigation would not only help understand the inhibitory properties of this unique compound class against human CYP enzymes but also advance our strategy in assessing DDI potential for other chemically reactive drug molecules, such as various covalent modulators, another novel chemical class of increasing interest in drug discovery and development.

\section{Materials and Methods}

Chemicals and Reagents. All reagents were in the highest grade commercially available. Bupropion, phenacetin, paclitaxel, $(S)$-(-)-warfarin, dextromethorphan, $(S)-(+)$-mephenytoin, ticlopidine, and NADPH were purchased from Sigma-Aldrich (St. Louis, MO). Midazolam, 1-hydroxy midazolam- $\left[{ }^{13} \mathrm{C} 3\right]$, human liver microsomes (mixed sex, 150 donors pooled), and Supersomes containing recombinant CYP (rCYP) 2B6 were all obtained from Corning
(Corning, New York). Acetaminophen- $\mathrm{D}_{4}$, phenyl- $\mathrm{D}_{5}-7$-hydroxywarfarin, hydroxybupropion- $\mathrm{D}_{6}, 4^{\prime}$-hydroxymephenytoin- $\mathrm{D}_{3}, 6 \alpha$-hydroxypaclitaxel- $\mathrm{D}_{5}$, and dextrorphan- $\mathrm{D}_{3}$ were purchased from Toronto Research Chemicals (North York, Ontario, Canada). Acetonitrile and formic acid were purchased from EMD Millipore (Hayward, CA). Glacial acetic acid was purchased from BDH Chemicals (London, England). Potassium phosphate, PBD, and the PBD analogs were obtained internally from Genentech compound management.

Reversible CYP Inhibition. CYP inhibition assay was performed in triplicate on a Tecan Freedom Evo (Morrisville, NC). Briefly, PBD dimer, PBD analogs, or positive controls in DMSO stock were serially diluted in phosphate buffer (KPi 10 $\mathrm{mM}, \mathrm{pH}$ 7.4) containing human liver microsomes (HLMs) and individual CYP isoform specific substrates. After brief warm-up at $37^{\circ} \mathrm{C}$, enzymatic reaction was initiated by adding $1 \mathrm{mM} \mathrm{NADPH}$ to HLM-substrate mixture. The final reaction mixtures contained $0.03 \mathrm{mg} / \mathrm{ml} \mathrm{HLM}$, a test compound (PBD or analog) at various concentrations $(0,0.0032,0.016,0.08,0.4,2$, and $10 \mu \mathrm{M})$, and individual CYPspecific substrates, including phenacetin $(50 \mu \mathrm{M}, 1 \mathrm{~A} 2)$, bupropion ( $80 \mu \mathrm{M}, 2 \mathrm{~B} 6)$, paclitaxel (4 $\mu \mathrm{M}, 2 \mathrm{C} 8), S$-(-)-warfarin $(2 \mu \mathrm{M}, 2 \mathrm{C} 9), S$-(+)-mephenytoin $(60 \mu \mathrm{M}$, 2C19), dextromethorphan (5 $\mu \mathrm{M}, 2 \mathrm{D} 6)$, and midazolam ( $2 \mu \mathrm{M}, \mathrm{CYP} 4 / 5)$. After incubation at $37^{\circ} \mathrm{C}$ for $10-30$ minutes, depending on the CYP-specific substrate, reaction was terminated with acetonitrile $(\mathrm{ACN})$ containing $6 \%$ acetic acid and internal standards. The samples were centrifuged at $2000 \mathrm{~g}$ for 10 minutes to remove precipitated protein; the resulting supernatants were transferred to a fresh plate and diluted with $0.1 \%$ formic acid water and subsequently were subjected to LC-MS/MS analysis for CYP-specific metabolites, including acetaminophen (1A2), hydroxybupropion (2B6), 6- $\alpha$-hydroxypaclitaxel (2C8), 7-hydroxywarfarin (2C9), (S)-(+)-4'-hydroxymephenytoin (2C19), dextrorphan (2D6), and 1hydroxymidazolam (CYP4/5). Stable isotope-labeled CYP-specific metabolites were used as internal standards. CYP activity was expressed as the peak area ratio of a CYP-specific metabolite relative to its internal standard, and percent inhibition was calculated from CYP activity of samples containing PBD relative to the solvent control (no PBD). Inhibition data were processed using Prism to calculate $\mathrm{IC}_{50}$ values for individual CYP enzymes.

Time-Dependent CYP Inhibition. The diluted method was used as previously described in literature (Parkinson et al., 2011) for TDI assessment using the same incubation conditions of the reversible inhibition assay. First, PBD dimer was serially diluted to $0,0.032,0.16,0.8,4,20$, and $100 \mu \mathrm{M}$ in phosphate buffer (pH 7.4) containing $0.3 \mathrm{mg} / \mathrm{ml} \mathrm{HLM}$, and two separate sets of preincubation were performed at $37^{\circ} \mathrm{C}$ for 30 minutes in the presence and absence of $1 \mathrm{mM} \mathrm{NADPH}$. After preincubation, aliquots of reaction mixture were transferred to a fresh plate and mixed with nine-volume of KPi buffer containing individual CYP-specific 
TABLE 1

Reversible inhibition of common CYPs in HLM by PBD

CYP inhibition assay was performed in triplicate as described under Materials and Methods. Individual CYP activity was expressed as the peak area ratio of a CYP-specific metabolite relative to its internal standard, and percent inhibition was calculated from CYP activity of samples containing PBD relative to the solvent control (no PBD). $\mathrm{IC}_{50}$ values of individual CYP enzymes were determined using Prism.

\begin{tabular}{lc}
\hline CYP & $\begin{array}{c}\text { Reversible CYP Inhibition } \\
\text { IC }_{50} \\
(\mu \mathrm{M})\end{array}$ \\
\hline $1 \mathrm{~A} 2$ & $>10$ \\
2B6 & $>10$ \\
2C8 & $1.1 \pm 0.3$ \\
2C9 & $>10$ \\
2C19 & $>10$ \\
2D6 & $>10$ \\
3A4/5 & $>10$ \\
\hline
\end{tabular}

substrates and $1 \mathrm{mM}$ NADPH, and incubation continued for additional 10-30 minutes, depending on the CYP-specific substrate, before quenching the incubation with ACN (6\% acetic acid) containing internal standards. Samples were processed and then analyzed as described in the reversible CYP inhibition assay. For each CYP enzyme, a pair of sigmoidal dose-response curves was generated to calculate two $\mathrm{IC}_{50}$ values, respectively, for both in the presence and absence of NADPH in the preincubation.

Inactivation Rate of CYP2B6 by PBD. PBD dimer $(2 \mu \mathrm{M})$ was initially incubated with $0.3 \mathrm{mg} / \mathrm{ml} \mathrm{HLM}$ in $100 \mathrm{mM}$ phosphate buffer ( $\mathrm{pH} \mathrm{7.4)}$ in the absence and presence of $1 \mathrm{mM}$ NADPH. At different time intervals, aliquots of reaction mixture were transferred and immediately mixed with nine-volume of KPi buffer containing both $1 \mathrm{mM}$ NADPH and bupropion $(80 \mu \mathrm{M})$, and then incubation continued for 15 minutes before quenching the incubation with $\mathrm{ACN}$ (6\% acetic acid) containing hydroxybupropion- $\mathrm{D}_{6}$ (IS). Resulting

samples were subjected to LC-MS/MS analysis for CYP2B6 activity as described above.

Irreversibility of CYP2B6 Inhibition by PBD. HLM was first diluted to $0.5 \mathrm{mg} / \mathrm{ml}$ in $100 \mathrm{mM} \mathrm{KPi}$ buffer and was divided into three portions (A, B, and C, $0.5 \mathrm{ml}$ each) in triplicate. After spiking $10 \mu \mathrm{M}$ PBD in portion $\mathrm{C}$, all three HLM samples (A, B, and C) were incubated for 60 minutes at $37^{\circ} \mathrm{C}$. At the end of the incubation, portion B was supplied with PBD dimer $(10 \mu \mathrm{M})$. Subsequently, all three samples were centrifuged at 10,000 RPM for 5 minutes using an Effendorf Microfuge 5424 to remove supernatants, and the resulting HLM pellets were then washed with $1 \mathrm{ml}$ PBS and followed by centrifugation to remove residual PDB dimer in supernatants. Finally, the treated HLM pellets were resuspended with $100 \mathrm{mM} \mathrm{KPi}$ with final HLM concentration of $0.05 \mathrm{mg} / \mathrm{ml}$ to measure CYP2B6 activity in $\mathrm{A}, \mathrm{B}$, and $\mathrm{C}$ by adding bupropion $(80 \mu \mathrm{M})$ and NADPH $(1 \mathrm{mM})$ as described in the above section. CYP2B6 activity in both $\mathrm{B}$ and $\mathrm{C}$ was expressed in relation to that in $\mathrm{A}$ (the control, $100 \%$ ).

Inhibition of rCYP2B6. PBD or its analogs were serially diluted in PBS buffer in triplicates and briefly warmed at $37^{\circ} \mathrm{C}$ after mixing with $\mathrm{NADPH}$ and bupropion. The reactions were initiated by the addition of rCYP2B6 in PBS. The reaction mixtures contained PBD $(0.003-10 \mu \mathrm{M}), 1 \mathrm{mM} \mathrm{NADPH}, 80 \mu \mathrm{M}$ bupropion, and $1.2 \mathrm{pmol} / \mathrm{ml} \mathrm{rCYP2B6}$ in a final volume of $100 \mu \mathrm{l}$ each. After 15 minutes incubation, the reaction mixtures were quenched with a final volume of $200 \mu \mathrm{l}$ of acetonitrile containing hydroxybupropion- $\mathrm{D}_{6}$ as the internal standard (IS). The samples were centrifuged at $2000 \mathrm{~g}$ for 10 minutes; the resulting supernatants were transferred to a 96-well sample plate, diluted with $0.1 \%$ formic acid water, and then subjected to LC-MS/MS analysis for CYP2B6 activity as described previously.

Time-Dependent Inhibition of rCYP2B6. Time-dependent inhibition of rCYP2B was performed similarly to the regular CYP TDI assay using HLM as described above. The only difference was replacing HLM with Supersomes containing rCYP2B6 (1.3 pmol/ml).

LC-MS/MS Method. The samples were analyzed using LC 1200SL Series System from Agilent (Santa Clara, CA) and an HTS PAL auto sampler from CTC

CYP2B6 Direct Inhibition

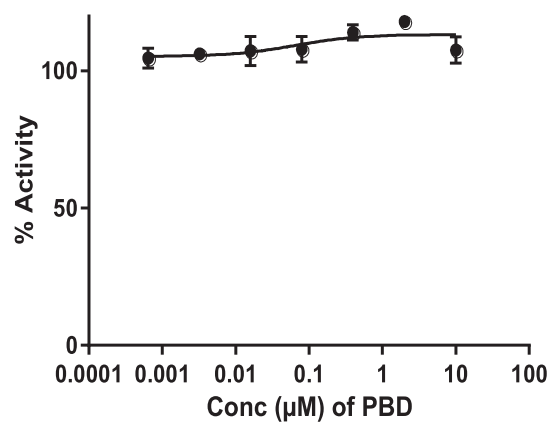

CYP2B6 Time-dependent

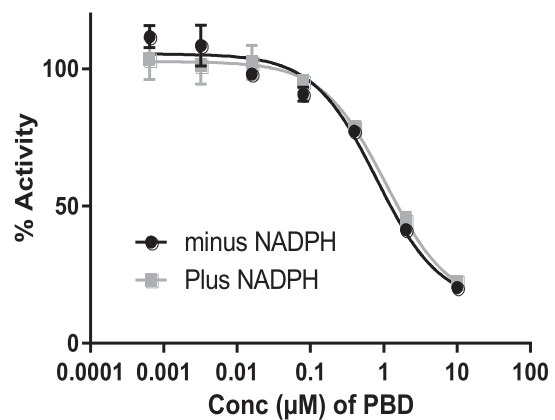

CYP2C8 Direct Inhibition

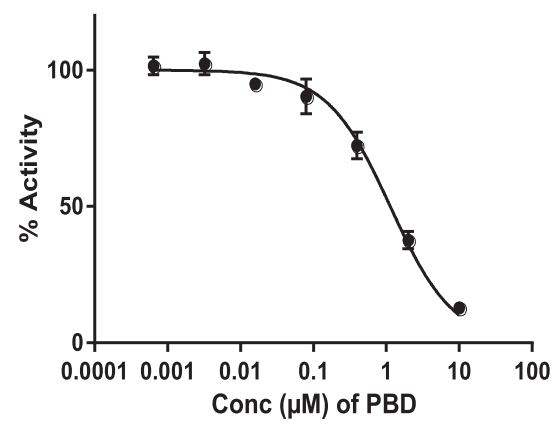

CYP2C8 Time-dependent

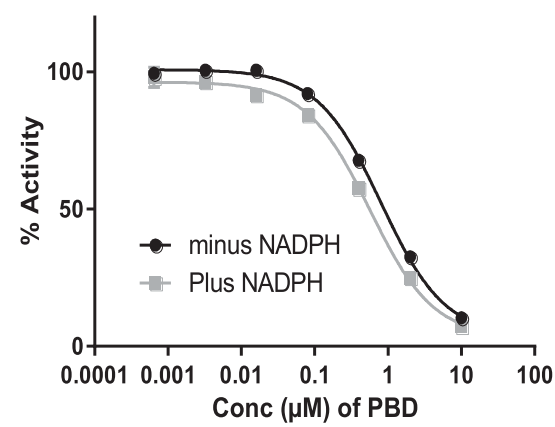

CYP3A4 Midazolam Direct Inhibition

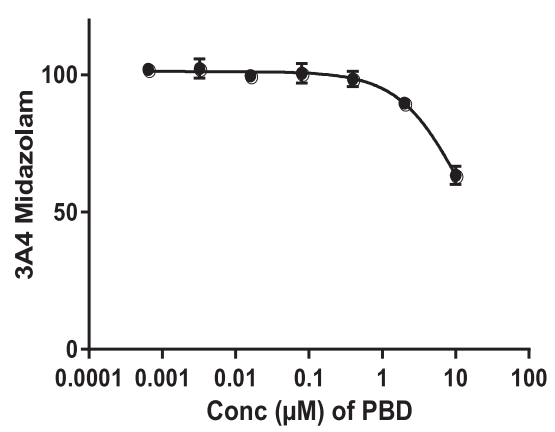

CYP3A4 Midazolam Time-dependent

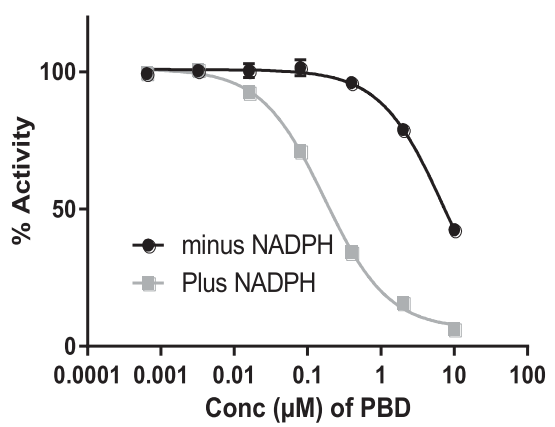

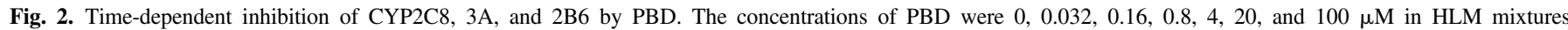

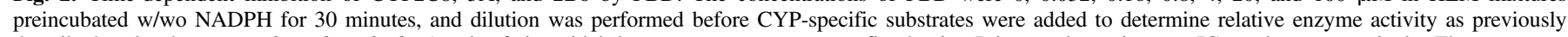

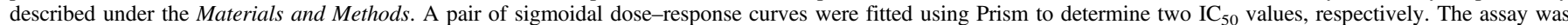
performed in triplicates. Conc, concentration. 
TABLE 2

TDI of common CYPs in HLM by PBD

The concentrations of PBD were $0,0.032,0.16,0.8,4,20$, and $100 \mu \mathrm{M}$ in HLM mixtures preincubated w/wo NADPH for 30 min, and a 10-fold dilution was performed before individual CYPspecific substrates were added to determine relative enzyme activity as previously described under Materials and Methods. The assay was performed in triplicates.

\begin{tabular}{|c|c|c|c|}
\hline \multirow[b]{2}{*}{ CYP } & \multicolumn{3}{|c|}{ TDI: $\mathrm{IC}_{50}(\mu \mathrm{M})$} \\
\hline & $\stackrel{-}{\text { NADPH }}$ & + NADPH & $\mathrm{IC}_{50}$ Ratio \\
\hline $1 \mathrm{~A} 2$ & $>10$ & $>10$ & NA \\
\hline $2 \mathrm{~B} 6$ & $1.5 \pm 0.07$ & $1.8 \pm 011$ & 0.8 \\
\hline $2 \mathrm{C} 8$ & $0.90 \pm 0.03$ & $0.6 \pm 0.02$ & 1.5 \\
\hline $2 \mathrm{C} 9$ & $>10$ & $>10$ & NA \\
\hline $2 C 19$ & $>10$ & $>10$ & NA \\
\hline 2D6 & $>10$ & $>10$ & NA \\
\hline $3 \mathrm{~A} 4 / 5$ & $7.5 \pm 0.18$ & $0.20 \pm 0.06$ & $>35$ \\
\hline
\end{tabular}

Analytics (Carrboro, NC) coupled with a 5500 QTRAP MS equipped with an electronspray ionization source. The separation of the analytes was performed using Luna Omega C18 columns $(1.6 \mu \mathrm{m}, 30 \times 2.1 \mathrm{~mm})$ from Phenomenex (Torrance, CA), and the analytical methods used are summarized in the Supplemental Material.

\section{Results}

Reversible Inhibition of CYP Activities by PBD in Human Liver Microsomes. The inhibitory effect of PBD on the activities of seven common CYP isozymes (CYP1A2, CYP2B6, CYP2C8, CYP2C9, CYP2C19, CYP2D6, and CYPCYP4/5) was assessed at microsomal protein concentration of $0.03 \mathrm{mg} / \mathrm{ml}$. As summarized in Table 1, PBD showed little or no inhibition for CYP1A2, CYP2B6, CYP2C9, CYP2C19, CYP2D6, and CYPCYP4/5 with $\mathrm{IC}_{50}$ greater than $10 \mu \mathrm{M}$ for all those isoforms. The only exception is inhibition of CYP2C8 with an $\mathrm{IC}_{50}$ value of $1.1 \pm 0.3 \mu \mathrm{M}$.

Time-Dependent Inhibition of CYP Activities by PBD in Human Liver Microsomes. The $\mathrm{IC}_{50}$ shift method was used to investigate the time-dependent inhibition properties of PBD in HLM. Similar to what was observed in the reversible inhibition assay, PBD showed little or no inhibition for CYP1A2, 2C9, 2C19, and 2D6 in the TDI assay, and no marked change in inhibition potency was observed between two preincubations with and without NADPH. For CYP3A4/5, 2C8, and 2B6, different inhibition profiles were observed (Fig. 2, A-C), and their $\mathrm{IC}_{50}$ shift patterns are summarized in Table 2. For CYP3A4/5 (Fig. 2A), PBD showed a significant potency shift, and its $\mathrm{IC}_{50}$ value decreased from $7.50 \mu \mathrm{M}$ without NADPH to $0.20 \mu \mathrm{M}$ with NADPH in the preincubation (Table 2); a 37-fold shift in $\mathrm{IC}_{50}$ indicates that $\mathrm{PBD}$ is a potent time-dependent CYP3A inhibitor. For CYP2C8, PBD showed nearly two identical inhibition profiles with and without NADPH (Fig. 2B), and the two corresponding $\mathrm{IC}_{50}$ values, 0.6 and $0.9 \mu \mathrm{M}$ (Table 2), are comparable to that $(1.1 \mu \mathrm{M})$ obtained in the reversible inhibition assay (Table 1), which suggests that PBD is not a time-dependent inhibitor of CYP2C8. Similar to that of CYP2C8, PBD exhibited two nearly identical inhibition profiles against CYP2B6 (Fig. 2C) and two comparable $\mathrm{IC}_{50}$ values $(1.50 \mu \mathrm{M}$ vs. $1.8 \mu \mathrm{M})$ (Table 2$)$, suggesting that PBD is not a timedependent inhibitor for CYP2B6. However, both values are markedly lower than the $\mathrm{IC}_{50}(>10 \mu \mathrm{M})$ obtained in the direct reversible inhibition assay.

NADPH-Independent CYP2B6 Inactivation. To further confirm NADPH-independent inactivation of CYP2B6, formation of hydroxybupropion was monitored at different incubation times in PBD-HLM mixtures in the absence and presence of NADPH. As shown in Fig. 3, remaining CYP2B6 activity in HLM decreased with incubation time, and the slope of activity loss over the time course is almost identical between two preincubations with and without NADPH.
Time-Dependent Inhibition of rCYP2B6 by PBD and Analogs. PBD and five different analogs (PBD1-5) were further tested for both reversible and time-dependent inhibition in Supersomes expressing CYP2B6. As shown in Table 3, PBD showed weak inhibition against rCYP2B6 in the direct reversible inhibition $\left(\mathrm{IC}_{50}>10 \mu \mathrm{M}\right)$, but potent inhibition was observed in the TDI assay in both the absence and presence of $\mathrm{NADPH}$ with $\mathrm{IC}_{50}$ of 1.5 and $0.2 \mu \mathrm{M}$, respectively. In contrast, none of those five analogs showed potent inhibition $(>10 \mu \mathrm{M})$ against CYP2B6 in both reversible inhibition and TDI assay.

Irreversibility of CYP2B6 Inhibition by PBD. To determine the reversibility of the inactivation of CYP2B6 by PBD, three different HLM incubations were performed separately: HLM only (A), HLM spiked with PBD after incubation (B), and HLM incubated with PBD (C), which was followed by centrifugation and washing to remove remaining PBD in HLM pellets (B and C) as described in the Materials and Methods. As shown in Fig. 4, HLM spiked with PBD after incubation (B) showed nearly the same level of CYP2B6 activity compared with that in HLM without PBD (control A), indicating that PBD was completely removed from the samples by centrifugation. In contrast, incubation of HLM with PBD (C) resulted in a loss of more than 30\% CYP2B6 activity compared with either one without PBD in the incubation (A) or the other spiked with PBD after incubation (B),

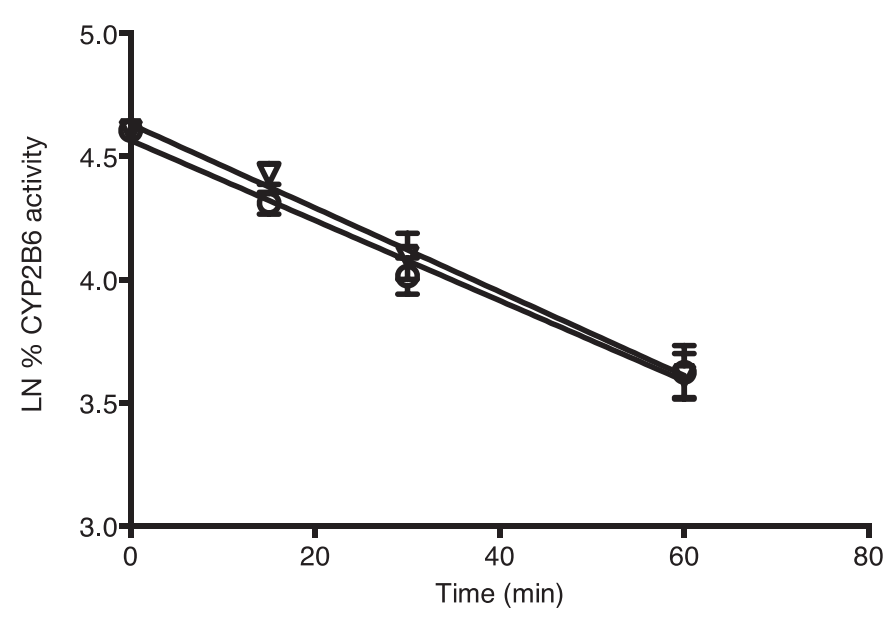

Fig. 3. Inactivation of CYP2B6 in HLM incubated with PBD in the absence of NADPH $(O)$ and presence of NADPH $(\Delta)$. PBD $(2 \mu \mathrm{M})$ was first incubated in triplicates with $0.3 \mathrm{mg} / \mathrm{ml}$ HLM w/wo NADPH. Aliquots of reaction mixtures were transferred at different time intervals, and immediately mixed with nine-volume of KPi buffer containing both NADPH and bupropion to determine CYP2B6 activity. CYP2B6 activity at different time points was expressed relative to that at T0 (100\%). CYP2B6 activity loss caused by thermo-inactivation was $16 \%$ approximately after 60 minutes incubation at $37^{\circ} \mathrm{C}$. LN: Natural $\log$ scale. 
TABLE 3

TDI of common CYP2B6 in HLM by PBD analogs

The concentrations of PBD and its analogs were $0,0.032,0.16,0.8,4,20$, and $100 \mu \mathrm{M}$ in HLM mixtures preincubated w/wo NADPH for 30 min, and then dilution was performed before individual CYP-specific substrates were added to determine relative enzyme activity as previously described under Materials and Methods.

\begin{tabular}{|c|c|c|c|c|}
\hline \multicolumn{5}{|c|}{ Recombinant CYP2B6 } \\
\hline Name & Structure & $\begin{array}{l}\text { Direct Inhibition } \\
\mathrm{IC}_{50}(\mu \mathrm{M})\end{array}$ & $\begin{array}{l}- \text { NADPH } \\
\mathrm{IC}_{50}(\mu \mathrm{M})\end{array}$ & $\begin{array}{l}\text { + NADPH } \\
\mathrm{IC}_{50}(\mu \mathrm{M})\end{array}$ \\
\hline PBD & & $>10$ & 1.5 & 0.2 \\
\hline PBD-2 & & $>10$ & $>10$ & $>10$ \\
\hline PBD-4 & & $>10$ & $>10$ & $>10$ \\
\hline
\end{tabular}

suggesting that removal of unbound PBD by centrifugation and washing did not lead to complete recovery of hydroxylase activity of PBD-treated samples.

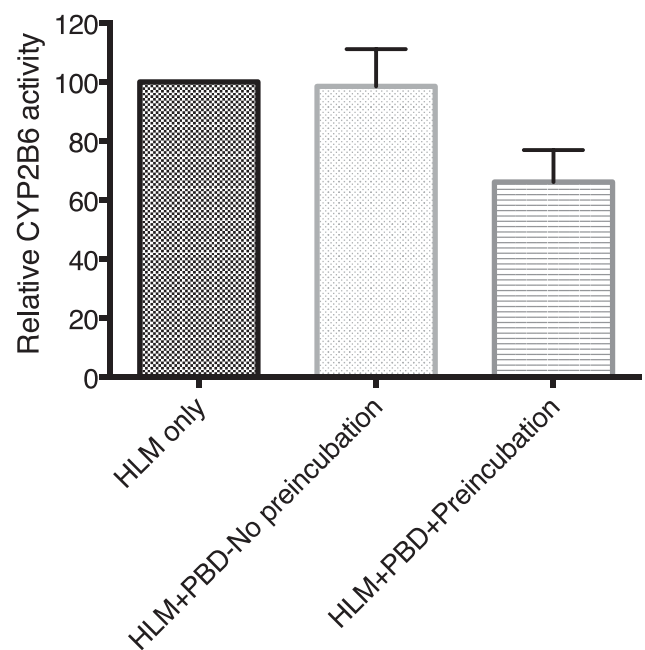

Fig. 4. Recovery of CYP2B6 activity in HLM incubated with PBD after washing. Three incubations were performed for 60 minutes at $37^{\circ} \mathrm{C}$ in triplicate: HLM alone (left bar), HLM +10 $\mu$ M PBD (right bar), and HLM spiked with PBD after incubation (middle bar). All samples were then centrifuged to pellet HLM, which was followed by washing with $1 \mathrm{ml}$ PBS to remove residual PDB. HLM pellets were resuspended with $100 \mathrm{mM}$ KPi to a final HLM concentration of $0.05 \mathrm{mg} / \mathrm{ml}$ to measure CYP2B6 activity as described under the Materials and Methods. CYP2B6 activity loss in HLM incubated with PBD (right bar) suggests that removal of unbound PBD by centrifugation and washing did not lead to complete recovery of hydroxylase activity of PBD-treated samples.

\section{Discussion}

CYP inhibition is regarded as one of the most common mechanisms leading to clinical DDIs. CYP inhibition is classified mechanistically as reversible and irreversible inhibition, and irreversible inhibition is usually caused by bioactivation catalyzed by a CYP enzyme, resulting in a reactive intermediate that tightly binds to the enzyme (Hollenberg et al., 2008). Thus, irreversible CYP inhibition is NADPH-dependent. Our results show that PBD is not an inhibitor for CYP1A2, 2C9, 2C19, and 2D6 up to $10 \mu \mathrm{M}$ but is a potent reversible inhibitor for CYP2C8 $\left(\mathrm{IC}_{50}=1.1 \mu \mathrm{M}\right)$. Additionally, PBD is a time-dependent inhibitor for CYPCYP4/5, as evidenced by a more than 30 -fold shift in $\mathrm{IC}_{50}$ observed in the TDI assay (Table 2). PBD is predominately metabolized by CYP3A4/5 in HLM (unpublished data). When incubated in HLM in the presence of GSH and NADPH, two glutathione conjugates were detected as a direct conjugate (PBD-GSH, $\mathrm{m} / \mathrm{z}$ 891.3475) and an oxidative conjugate (PBD-O-GS, m/z 907.3425), respectively (unpublished data), suggesting that reactive intermediates were formed in HLM incubation. Therefore, it is reasonable to speculate that those reactive intermediates likely bind and inactivate CYP3A4/5, although involvement of other CYPs is also possible.

Interestingly, PBD did not inhibit CYP2B6 in the reversible inhibition assay but showed potent inhibition of CYP2B6 in the TDI assay w/wo $\mathrm{NADPH}\left(\mathrm{IC}_{50}\right.$ of $1.5 \mu \mathrm{M}$ vs. $\left.1.8 \mu \mathrm{M}\right)$. Although no marked shift in $\mathrm{IC}_{50}$ was observed in TDI assay, a more than 5-fold decrease in $\mathrm{IC}_{50}$ was observed between TDI and reversible inhibition assay, and the shift in potency is unlikely due to the difference in $f_{u \text {,mic }}$ since HLM concentration was the same in both assays; additionally, highly comparable $\mathrm{IC}_{50}$ values were obtained for CYP2C8 between the same two assays $(1.1 \pm 0.3 \mu \mathrm{M}$ vs. $0.9 \pm 0.03 \mu \mathrm{M})$, suggesting that a decrease in $\mathrm{IC}_{50}$ of CYP2B6 was not caused by different incubation 

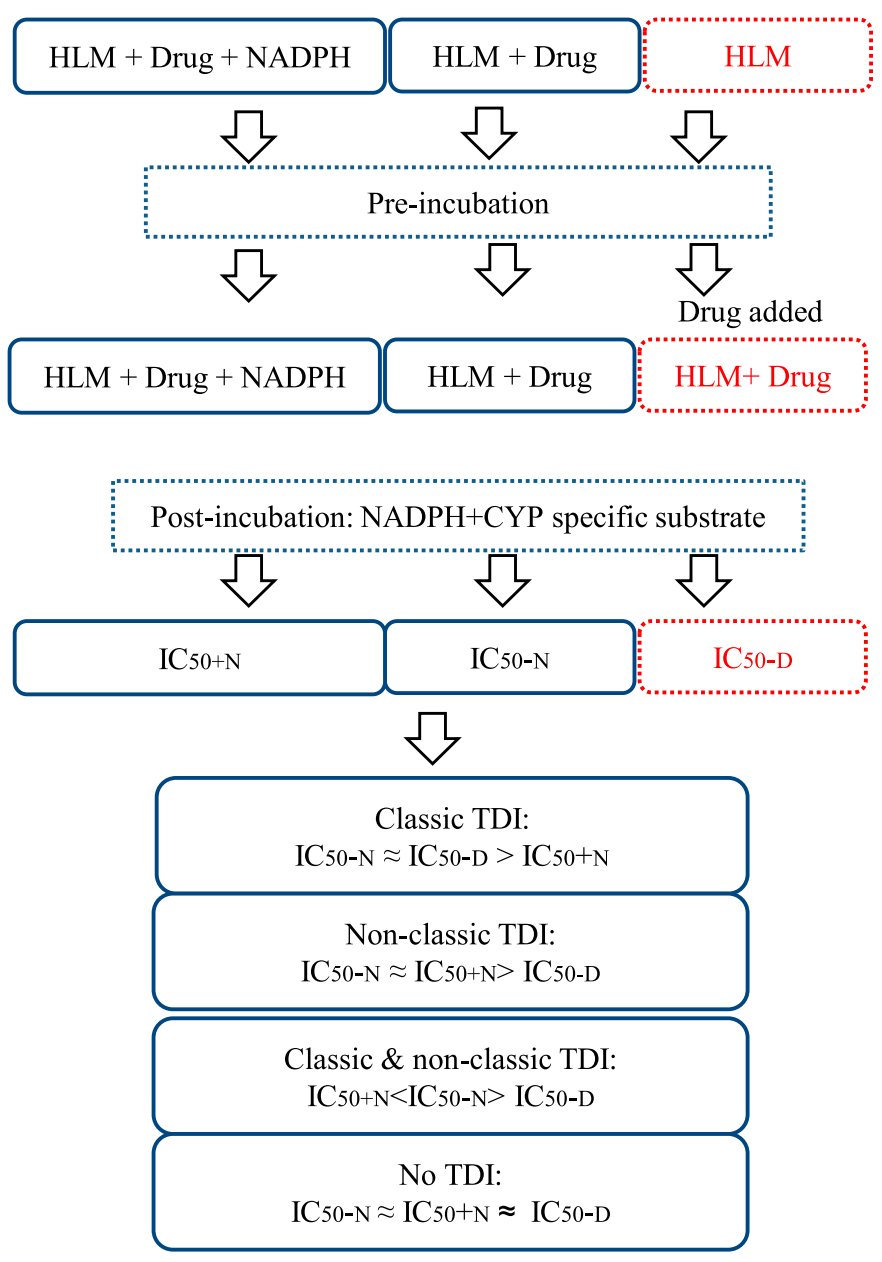

Fig. 5. A revised TDI assay setup for reactive modulators. Three preincubations are performed: HLM + drug (a, left), HLM + drug + NADPH (b, middle), and HLM alone (c, right). After preincubation, the drug is spiked to incubation sample c. Subsequently, incubation mixtures are diluted and then assayed for CYP-specific activity to obtain three $\mathrm{IC}_{50}$ values, respectively: $\mathrm{IC}_{50-\mathrm{N}}(\mathrm{a}$, without $\mathrm{NADPH}), \mathrm{IC}_{50+\mathrm{N}}$ (b, with $\mathrm{NADPH}$ ), and $\mathrm{IC}_{50-\mathrm{D}}(\mathrm{c}$, without drug in preincubation).

conditions. Thus, we concluded that inactivation of CYP2B6 by PBD in HLM is NADPH-independent. This conclusion is supported by the observation that the rate of CYP2B6 inactivation in HLM-PBD mixtures is almost identical w/wo NADPH in preincubation (Fig. 3). Additionally, NADPH-independent inhibition of CYP2B6 by PBD seems irreversible since removal of PBD from HLM mixtures did not fully restore enzymatic activity (Fig. 4).

NADPH-independent inactivation of CYP2B6 by PBD was further confirmed in Supersomes containing rCYP2B6, which showed a more than 5 -fold decrease in $\mathrm{IC}_{50}$ in the absence of NADPH compared with that measured in the reversible inhibition assay. Interestingly, PBD is more potent in the presence of NADPH $\left(\mathrm{IC}_{50}\right.$ $=0.2 \mu \mathrm{M})$ compared with that in the absence of NADPH $\left(\mathrm{IC}_{50}=\right.$ $1.5 \mu \mathrm{M}$ ), a 7 -fold shift in $\mathrm{IC}_{50}$ raising the possibility that, in addition to NADPH-independent inactivation, CYP2B6 was also inactivated by PBD via NADPH-dependent bioactivation. Apparently, NADPH-dependent inactivation of CYP2B6 seemed unnoticeable in HLM, as indicated by two comparable $\mathrm{IC}_{50}$ values (1.8 $\mu \mathrm{M}$ vs. $1.5 \mu \mathrm{M})$ measured in TDI assay w/wo NADPH, respectively; additionally, in TDI assay, $\mathrm{PBD}$ appeared to be more potent against CYP2B6 in Supersomes $\left(\mathrm{IC}_{50}=0.2 \mu \mathrm{M}\right)$ than HLM $\left(\mathrm{IC}_{50}=1.8 \mu \mathrm{M}\right)$ when NADPH was present in preincubation. Both differences in inhibition potency observed between Supersomes and HLM might be explained by potential metabolism of PBD by CYP3A4/5, leading lower PBD concentrations in HLM than in Supersomes.

Approximately, CYP2B6 accounts for 2\%-10\% of total hepatic P450 content, and it metabolizes 3\%-12\% of all drugs (Zanger et al., 2007; Wang and Tompkins, 2008). So far, several CYP2B6 TDI inhibitors have been reported including isopsoralen ( $\mathrm{Lu}$ et al., 2016), isoimperatorin (Cao et al., 2015), selegiline (Sridar et al., 2012), ticlopidine, and clopidogrel (Richter et al., 2004). Compared with those known TDI inhibitors, PBD is highly unusual given that inactivation of CYP2B6 does not require NADPH-dependent bioactivation. Presumably, this uncharacteristic TDI is attributed to the fact that PBD contains two imines that can chemically react with both thiol and amines of different amino acid residues, resulting in covalent binding and subsequent inactivation of the enzyme without CYP2B6-mediated bioactivation of PBD. This assumption is quite reasonable since it has been wellestablished that two imines of PBD can chemically react with the $\mathrm{C}_{2}$ $\mathrm{NH}_{2}$ groups of guanine bases of the targeted DNA to form a covalent aminal bond (Hartley, 2011; Mantaj et al., 2017). Consistently, PBD-1, a highly similar analog containing two amines instead of imines, did not show any TDI under the same assay conditions.

It is apparent that inactivation of CYP2B6 is not simply due to random chemical reactions of PBD with the enzyme, since PBD-4, a monomer analog containing one reactive imine group, did not inactivate CYP2B6. Additionally, it is important to note that, PBD-2 and PBD-3, two structurally similar analogs containing only one imine at different locations, did not exhibit TDI against CYP2B6, suggesting that formation of two distinct covalent bonds is likely required to stabilize the PBD-enzyme complex, which is consistent with the fact that PBD forms a covalent aminal bond only after the molecule is secured within the minor groove even though the major DNA major-groove also contains guanine bases (Mantaj et al., 2017). Therefore, we hypothesized that PBD must first associate with CYP2B6 reversibly to bring two reactive imine groups within close proximity of their corresponding amino acid residues of the enzyme to form two covalent bonds, resulting in a stable and inactive PBD-enzyme complex.

CYP2B6 contains multiple cysteine residues and a number of arginine and lysine residues with amine groups (Mo et al., 2009). It is difficult to postulate how exactly PBD binds to the enzyme. Treatments of recombinant CYP2B6 with PBD followed by gel electrophoresis/trypsin digestion and subsequent LC-MS/MS analysis achieved more than $85 \%$ sequence coverage but failed to locate the binding sites despite repeated attempts. One possible explanation for the failure is the reversible nature of aminal bonding between PBD and CYP2B6, which may require the intact PBD-protein complex to be stabilized, and such postulation is substantiated by our finding that no putative adducts were detected by liquid chromatography-mass spectrometry after PBD was incubated with free guanine or lysine. Alternatively, it is possible that PBD-peptide complexes derived from trypsin digestion are not chemically stable under experimental conditions.

PBD dimer was designed to be a DNA minor-groove covalent binder (Hartley et al., 2004; Zhang et al., 2016), and no protein covalent binding is known so far. In fact, PBD dimer was nearly recovered completely $(>95 \%)$ after 24-hour incubations with human and mouse plasma (unpublished data), indicating its low potential for protein covalent binding. Inactivation of CYP2B6 through protein covalent binding is a new finding, although the chemical nature of the covalent binding is not known between PBD and the enzyme. The adduct formation of PBD dimer with GSH in buffer suggested addition of thiol to an imine. There are limited examples that demonstrate the chemical-binding properties of reactive intermediates or drugs to endogenous proteins. Covalent binding of acetaminophen to proteins was through cysteine thiol group 
connecting to quinone imine intermediate (Leeming et al., 2015). MaxiPost formed an irreversible covalent bond with the $\varepsilon$-amino group of a lysine residue of albumins (Zhang et al., 2005). Acrylamidecontaining covalent inhibitors, such as neratinib, osimertinib, and ibrutinib, are intrinsically reactive to endogenous proteins. For example, neratinib was found to form covalent adducts on Lys-190 of human serum albumin (Wang et al., 2010).

Screening for TDI is commonly achieved by comparing two $\mathrm{IC}_{50}$ values determined w/wo NADPH (Riley et al., 2007). As shown in this study, drug candidates with reactive moieties can directly inactivate CYPs in an NADPH-independent manner, resulting in two comparable $\mathrm{IC}_{50}$ values and false positives in the TDI assay. Therefore, a revised TDI assay is proposed specifically for compounds with reactive groups. As shown in Fig. 5, in addition to two preincubations w/wo NADPH, a third incubation without the drug is included; after preincubation, the drug is added to the third preincubation, resulting in three $\mathrm{IC}_{50}$ values, respectively: $\mathrm{IC}_{50-\mathrm{N}}$ (without $\mathrm{NADPH}$ ), $\mathrm{IC}_{50+\mathrm{N}}$ (with $\mathrm{NADPH}$ ), and $\mathrm{IC}_{50-\mathrm{D}}$ (without drug). For NADPH-dependent TDI, IC $\mathrm{I}_{50-\mathrm{N}}$ and $\mathrm{IC}_{50-\mathrm{D}}$ will be comparable and both values should be higher than $\mathrm{IC}_{50+\mathrm{N}}$ because of inactivation of $\mathrm{CYP}$ in preincubation; for NADPHindependent $\mathrm{TDI}$, both $\mathrm{IC}_{50-\mathrm{N}}$ and $\mathrm{IC}_{50+\mathrm{N}}$ are lower than $\mathrm{IC}_{50-\mathrm{D}}$ because of direct inactivation of CYP enzyme by the reactive drug. If the CYP enzyme is inactivated by both reaction intermediates and the reactive moiety of the drug, one should expect that $\mathrm{IC}_{50+\mathrm{N}}$ will be lower than $\mathrm{IC}_{50-\mathrm{N}}$, and both values will be lower than $\mathrm{IC}_{50-\mathrm{D}}$. NADPH-independent and NADPH-dependent TDI are ruled out if three $\mathrm{IC}_{50}$ values are in agreement.

In conclusion, we have shown a unique CYP inhibition profile of PBD: a potent reversible CYP2C8 inhibitor, an NADPH-dependent CYP3A4/5 TDI inhibitor, and an NADPH-independent CYP2B6 TDI inhibitor. To the best of our knowledge, the present study is the first example showing three types of CYP inhibition through different mechanisms: reversible drug-enzyme binding, enzyme inactivation via bioactivation, and enzyme inactivation by covalent binding via chemical reactions. Because of the unique nature of covalent modulators, a revised TDI assay is advised to avoid false positives.

\section{Acknowledgments}

The authors would like to thank Susan Wong for critical comments.

\section{Authorship Contributions}

Participated in research design: Kosaka, Zhang, Wong, Yan.

Conducted experiments: Kosaka.

Performed data analysis: Kosaka, Zhang, Wong, Yan.

Wrote or contributed to the writing of the manuscript: Kosaka, Zhang, Yan.

\section{References}

Cao J, Zheng L, Ji L, Lu D, Peng Y, and Zheng J (2015) Mechanism-based inactivation of cytochrome P450 2B6 by isoimperatorin. Chem Biol Interact 226:23-29.

Cipolla L, Araújo AC, Airoldi C, and Bini D (2009) Pyrrolo[2,1-c][1,4]benzodiazepine as a scaffold for the design and synthesis of anti-tumour drugs. Anticancer Agents Med Chem 9:1-31.

Gregson SJ Howard PW, et al. (2004) Linker length modulates DNA crosslinking reactivity and cytotoxic potency of $\mathrm{C} 8 / \mathrm{C}^{\prime}$ ether-linked C2-exo-unsaturated pyrrolo[2,1-c][1,4]benzodiazepine (PBD) dimers. J Med Chem 47:1161-1174.

Hartley JA (2011) The development of pyrrolobenzodiazepines as antitumour agents. Expert Opin Investig Drugs 20:733-744.
Hollenberg PF, Kent UM, and Bumpus NN (2008) Mechanism-based inactivation of human cytochromes p450s: experimental characterization, reactive intermediates, and clinical implications. Chem Res Toxicol 21:189-205.

Kotecha M Kluza J, et al. (2008) Inhibition of DNA binding of the NF-Y transcription factor by the pyrrolobenzodiazepine-polyamide conjugate GWL-78.. Mol Cancer Ther 7:1319-1328.

Leeming MG, Gamon LF, Wille U, Donald WA, and O'Hair RA (2015) What are the potential sites of protein arylation by N-Acetyl-p-benzoquinone imine (NAPQI)? Chem Res Toxicol 28 2224-2233.

Lu D, Ji L, Zheng L, Cao J, Peng Y, and Zheng J (2016) Mechanism-based inactivation of cytochrome P450 2B6 by isopsoralen. Xenobiotica 46:335-341.

Mantaj J, Jackson PJM, Rahman KM, and Thurston DE (2017) From anthramycin to pyrrolobenzodiazepine (PBD)-containing antibody-drug conjugates (ADCs). Angew Chem Int Ed Engl 56:462-488.

Martin C, Ellis T, McGurk CJ, Jenkins TC, Hartley JA, Waring MJ, and Thurston DE (2005) Sequence-selective interaction of the minor-groove interstrand cross-linking agent SJG-136 with naked and cellular DNA: footprinting and enzyme inhibition studies. Biochemistry 44 (11): 4135-4147.

Mo SL, Liu YH, Duan W, Wei MQ, Kanwar JR, and Zhou SF (2009) Substrate specificity, regulation, and polymorphism of human cytochrome P450 2B6. Curr Drug Metab 10 730-753.

Parkinson A, Kazmi F, Buckley DB, et al. (2011) An evaluation of the dilution method for identifying metabolism-dependent inhibitors of cytochrome P450 enzymes. Drug Metab Dispos Drug Metab Dispos 39:1370-1387 (39):1370-1387.

Phillips T, Barr PM, Park SI, Kolibaba K, Caimi PF, Chhabra S, Kingsley EC, Boyd T, Chen R, Carret AS, et al. (2019) A phase 1 trial of SGN-CD70A in patients with CD70positive diffuse large B cell lymphoma and mantle cell lymphoma. Invest New Drugs 37: 297-306

Puvvada MS, Forrow SA, Hartley JA, Stephenson P, Gibson I, Jenkins TC, and Thurston DE (1997) Inhibition of bacteriophage T7 RNA polymerase in vitro transcription by DNA-binding pyrrolo [2,1-c][1,4]benzodiazepines. Biochemistry 36:2478-2484.

Puvvada MS, Hartley JA, Jenkins TC, and Thurston DE (1993) A quantitative assay to measure the relative DNA-binding affinity of pyrrolo[2,1-c] $[1,4]$ benzodiazepine (PBD) antitumour antibiotics based on the inhibition of restriction endonuclease BamHI. Nucleic Acids Res 21 (16):3671-3675.

Reid JM Buhrow SA, et al. (2011) Pharmacokinetics, pharmacodynamics and metabolism of the dimeric pyrrolobenzodiazepine SJG-136 in rats. Cancer Chemother Pharmacol 68 $777-786$.

Richter T, Mürdter TE, Heinkele G, Pleiss J, Tatzel S, Schwab M, Eichelbaum M, and Zanger UM (2004) Potent mechanism-based inhibition of human CYP2B6 by clopidogrel and ticlopidine. J Pharmacol Exp Ther 308:189-197.

Riley RJ, Grime K, and Weaver R (2007) Time-dependent CYP inhibition. Expert Opin Drug Metab Toxicol 3:51-66.

Shameem M Kumar R, et al. (2015) Synthetic modified pyrrolo[1,4] benzodiazepine molecules demonstrate selective anticancer activity by targeting the human ligase 1 enzyme: an in silico and in vitro mechanistic study. Chem-Biol Interact 237:115-124.

Sridar C, Kenaan C, and Hollenberg PF (2012) Inhibition of bupropion metabolism by selegiline: mechanism-based inactivation of human CYP2B6 and characterization of glutathione and peptide adducts. Drug Metab Dispos 40:2256-2266.

Wang H and Tompkins LM (2008) CYP2B6: new insights into a historically overlooked cytochrome P450 isozyme. Curr Drug Metab 9:598-610

Wang J, Li-Chan XX, Atherton J, Deng L, Espina R, Yu L, Horwatt P, Ross S, Lockhead S, Ahmad S, et al (2010) Characterization of HKI-272 covalent binding to human serum albumin. Drug Metab Dispos 38:1083-1093.

Zammarchi F, Corbett S, Adams L, Tyrer PC, Kiakos K, Janghra N, Marafioti T, Britten CE, Havenith CEG, Chivers S, et al. (2018) ADCT-402, a PBD dimer-containing antibody drug conjugate targeting CD19-expressing malignancies. Blood 131:1094-1105.

Zanger UM Klein K, et al. (2007) Polymorphic CYP2B6: molecular mechanisms and emerging clinical significance. Pharmacogenetics 8:743-759.

Zhang D, Dragovich PS, Yu SF, Ma Y, Pillow TH, Sadowsky JD, Su D, Wang W, Polson A, Khojasteh SC, et al. (2019) Exposure-efficacy analysis of antibody-drug conjugates delivering an excessive level of payload to tissues. Drug Metab Dispos 47:1146-1155.

Zhang D, Krishna R, Wang L, Zeng J, Mitroka J, Dai R, Narasimhan N, Reeves RA, Srinivas SR, and Klunk LJ (2005) Metabolism, pharmacokinetics, and protein covalent binding of radiolabeled Maxipost (BMS-204352) in humans. Drug Metab Dispos 33:83-93.

Zhang D, Pillow TH, Ma Y, Cruz-Chuh JD, Kozak KR, Sadowsky JD, Lewis Phillips GD, Guo J, Darwish M, Fan P, et al. (2016) Linker immolation determines cell killing activity of disulfide-linked pyrrolobenzodiazepine antibody-drug conjugates. ACS Med Chem Lett 7: 988-993.

Zheng B, Yu SF, Del Rosario G, Leong SR, Lee GY, Vij R, Chiu C, Liang WC, Wu Y, Chalouni C, et al. (2019) An anti-CLL-1 antibody-drug conjugate for the treatment of acute myeloid leukemia. Clin Cancer Res 25 (4):1358-1368.

Address correspondence to: Dr. Zhengyin Yan, Drug Metabolism and Pharmacokinetics, Genentech, Inc, 1 DNA Way, South San Francisco, CA 94080. E-mail: yanz7@gene.com 